\title{
EVOLUTION OF THE MAGNETIC HELICITY FLUX DURING THE FORMATION AND ERUPTION OF FLUX ROPES
}

\author{
P. Romano ${ }^{1}$, F. P. Zuccarello $2,3,4$, S. L. Guglielmino ${ }^{5}$, and F. ZucCarello ${ }^{5}$ \\ ${ }^{1}$ INAF-Osservatorio Astrofisico di Catania, Via S. Sofia 78, I-95123 Catania, Italy; paolo.romano@ oact.inaf.it \\ ${ }^{2}$ Centre for Mathematical Plasma-Astrophysics, KU Leuven, Celestijnenlaan 200B, B-3001 Leuven, Belgium \\ ${ }^{3}$ Fonds Wetenschappelijk Onderzoek Vlaanderen, Egmontstraat 5, B-1000 Brussels, Belgium \\ ${ }^{4}$ LESIA, Observatoire de Paris, CNRS, UPMC, Univ. Paris Diderot, 5 place Jules Janssen, F-92190 Meudon, France \\ ${ }^{5}$ Dipartimento di Fisica e Astronomia-Sezione Astrofisica, Università di Catania, Via S. Sofia 78, I-95123 Catania, Italy \\ Received 2014 May 21; accepted 2014 August 23; published 2014 September 30
}

\begin{abstract}
We describe the evolution and the magnetic helicity flux for two active regions (ARs) since their appearance on the solar disk: NOAA 11318 and NOAA 11675. Both ARs hosted the formation and destabilization of magnetic flux ropes. In the former AR, the formation of the flux rope culminated in a flare of $\mathrm{C} 2.3 \mathrm{GOES}$ class and a coronal mass ejection (CME) observed by Large Angle and Spectrometric Coronagraph Experiment. In the latter AR, the region hosting the flux rope was involved in several flares, but only a partial eruption with signatures of a minor plasma outflow was observed. We found a different behavior in the accumulation of the magnetic helicity flux in the corona, depending on the magnetic configuration and on the location of the flux ropes in the ARs. Our results suggest that the complexity and strength of the photospheric magnetic field is only a partial indicator of the real likelihood of an AR producing the eruption of a flux rope and a subsequent CME.
\end{abstract}

Key words: Sun: chromosphere - Sun: corona - Sun: flares - Sun: magnetic fields - Sun: photosphere

Online-only material: animation, color figures

\section{INTRODUCTION}

The term magnetic flux rope (FR) indicates a particular field topology characterized by a set of magnetic field lines that collectively wrap around a central, axial field line. Such a magnetic configuration is considered crucial in many models of the formation and eruption of solar prominences (Rust 1994; Low 1996). When FR configurations are incorporated in magnetohydrodynamics (MHD) models, the results are in agreement with the observations of the so-called three-part structure of coronal mass ejections (CMEs), as well as with their precursors (see Zuccarello et al. 2012). From the theoretical point of view, the origin of FRs is controversial. Some MHD simulations suggest that rising magnetic flux tubes require a minimum amount of twist to be able to rise cohesively through the convection zone (see, e.g., Emonet \& Moreno-Insertis 1998; Fan et al. 1998; Gibson et al. 2004). This is also confirmed by some photospheric observations (Tanaka 1991; Lites et al. 1995; Leka et al. 1996) that suggest the emergence of pre-twisted FRs. However, more recent numerical simulations have shown that an FR may not emerge bodily from below the photosphere, but reforms in the corona, i.e., the field lines wrap around a new central axis that is different from the original flux tube axis (Magara 2006; Fan 2009; Archontis et al. 2014). Moreover, the question of how the eruption of an FR is initiated and driven is still under debate (see Schmieder et al. 2013 and references therein).

The FR configuration can also explain observations of the socalled sigmoids, i.e., S-shaped loops, observed in corona in EUV and X-ray (Rust \& Kumar 1994). The sigmoids usually indicate sheared and twisted magnetic field configurations, which carry a field-aligned current and thus free magnetic energy (Jiang et al. 2014). They usually appear above a curved polarity inversion line (PIL).

Taking into account that the presence of FRs is usually accompanied by observations of shearing or rotating motions of magnetic features at the photospheric level (Brown et al. 2003) and that FRs are characterized by a helical configuration of the magnetic field, it is useful to measure the complexity and instability of the magnetic field by considering the magnetic helicity evolution in corona during the FR lifetime (Berger \& Field 1984; Démoulin \& Pariat 2009).

In this paper, we study the flux of magnetic helicity during the phase of formation of such structures in two active regions (ARs) characterized by a completely different magnetic configuration: AR NOAA 11318 (hereinafter AR 318) and AR NOAA 11675 (hereinafter AR 675). The comparison of these two targets, from the very beginning of their appearance on the solar disk, allows us to better understand the behavior of this important physical quantity over time, and it is useful to shed light on the relationship between FRs and CMEs. In Section 2, we describe the different data sets used for our study, while, in Section 3, we describe the global evolution of the two ARs with particular attention to the phases of FR formation and eruption. The results are presented in Section 4 and discussed in Section 5.

\section{OBSERVATIONS}

In order to investigate the chromospheric and coronal evolution of the selected ARs, we used images acquired by the Atmospheric Imaging Assembly (AIA; Lemen et al. 2012) on board the Solar Dynamic Observatory (SDO; Pesnell et al. 2012) with a pixel resolution of about 0.6 and a cadence of $12 \mathrm{~s}$.

For AR 318, we used images acquired between 2011 October 11 at 19:12 UT and October 15 at 23:48 UT, while, for AR 675, we used images acquired between 2013 February 16 at 00:00 UT and February 19 at 13:36 UT. AIA passbands at $335 \AA, 304 \AA$, and $193 \AA$ have been used for this study.

The investigation at chromospheric and coronal levels has been complemented by continuum intensity images and line-of-sight (LOS) magnetograms acquired by the Heliospheric 
and Magnetic Imager (HMI; Schou et al. 2012) at $6173 \AA$ with a pixel resolution of 0.5 and a temporal cadence of 12 minutes.

To analyze the magnetic evolution of the ARs during these time intervals, we also used SDO/HMI Space-weather Active Region Patches (SHARPs) data (Hoeksema et al. 2014), with the same pixel resolution and temporal cadence of the HMI continuum data set. These data series provide maps of the photospheric vector magnetic field and its uncertainty for the entire lifetime of the AR. The vector field is computed using the Very Fast Inversion of the Stokes Vector code (Borrero et al. 2011), which performs a Milne-Eddington inversion of the observed Stokes profiles, optimized for the HMI pipeline; the remaining $180^{\circ}$ azimuth ambiguity is resolved with the minimum energy code (Metcalf 1994). More details about the SHARP pipeline are reported in Bobra et al. (2014). The SHARP data have been corrected for the rotation angle of $180^{\circ}$ of HMI data and the vector magnetic field components have been transformed into the local solar frame, according to Gary \& Hagyard (1990), as described in Sun (2013).

Finally, to complement the front view of AR 675 around the time of the occurrence of an M1.9 class flare, we used data from the COR1 coronagraph (Thompson et al. 2003; Thompson \& Reginald 2008) on board the twin Solar Terrestrial Relations Observatory (STEREO; Kaiser et al. 2008) spacecraft. On the day of the M1.9 flare, the separation angles between Earth and STEREO-A and STEREO-B were $130^{\circ}$ and $138^{\circ}$, respectively. Since the AR was located at a longitude of about $20^{\circ} \mathrm{E}$, the partial eruption associated with the M1.9 flare was entirely obscured by the limb of the Sun from the vantage point of STEREO-A. Therefore, we only used the observations provided by STEREO-B. In particular, we used images acquired on 2013 February 17 by COR1-B with a pixel resolution of about $15^{\prime \prime}$ with a time cadence of 5 minutes.

\section{EVOLUTION OF ACTIVE REGIONS}

\section{1. $A R 318$}

As seen in the photosphere, AR 318 emerged close to the central meridian (N21E17) on 2011 October 11 at around 17:30 UT as a system of several pores characterized by alternating magnetic polarities that later on-on October 12 at about 17:30 UT - merged, forming two separate groups of pores. During the evolution of the AR, the preceding group of pores merged, eventually resulting in the formation of a sunspot characterized by an asymmetric penumbra as shown in Figure 1(A). As can be deduced from the figure, the following group of pores was quite fragmented (see Figures $1(B)$ and (C)). Just before the filament eruption and the associated C2.3 flare, on October 15 at 04:19 UT, the following group of pores disappeared and only an isolated pore was visible (Figure 1(D)).

Soon after the initial stages of the emergence process, during which several alternating polarities were visible, AR 318 displayed a simple bipolar configuration. Figure 1(E) shows the magnetic configuration of the AR during the main phase of the emergence, i.e., on October 13 at 05:59 UT. As can be deduced from this figure, the preceding sunspot was characterized by a negative polarity, while the following pores displayed a positive magnetic field. The magnetogram also shows the presence of the so-called magnetic tongues, often assumed to be evidence of emerging twisted magnetic flux tubes (Hood et al. 2009; Luoni et al. 2011). Figures $1(\mathrm{~F})-(\mathrm{H})$ show the subsequent evolution of the AR 318, up to the moment of eruption. The two magnetic polarities lose part of their compactness and display a quite elongated structure. Such a transition of the magnetic polarities from compact to elongated is often observed in numerical MHD simulations of emerging twisted flux tubes (e.g., Archontis \& Hood 2010).

The chromospheric evolution of the AR as deduced from AIA $304 \AA$ is shown in Figures $1(\mathrm{I})-(\mathrm{L})$. During the main phase of flux emergence, east-west directed dark threads connecting the bright facular regions associated with the compact polarities are visible. They resemble an arch filament system usually observed during the emergence of magnetic flux (e.g., Spadaro et al. 2004). Later on, contemporaneously to the transition from compact to elongated polarities, we observe the formation of a filament within the facular region. About $8 \mathrm{hr}$ before eruption, i.e., on October 14 at 20:20 UT (Figure 1(K)), the filament is clearly visible and displays a forward-S shape that suggests that the AR is characterized by a positive magnetic helicity (Démoulin \& Pariat 2009). The eruption of the filament and the associate C2.3 flare is shown in Figure 1(L). The CME time correlated with this flare has been observed by Large Angle and Spectrometric Coronagraph Experiment (LASCO) starting from 5:48 UT (see Figures 2(A) and (B)).

Finally, Figures 1(M)-(P) show the coronal evolution of the AR 318 as seen in the AIA $193 \AA$ passband. In the early stages of the emergence process, a system of loops displaying an arcadelike configuration and connecting the two opposite polarities is observed. However, while the emergence process continues, this loop system undergoes a transition from an arcade-like to a sigmoid-like configuration. Figure $1(\mathrm{O})$ shows the filament embedded in the forward-S shaped sigmoid. The transition from arcade-like to sigmoid-like configuration during the emergence process, the formation of the filament within the sigmoid, and the absence of a significant pre-existing magnetic field, strongly suggest that we are observing the emergence of a rather isolated twisted FR, that subsequently erupted.

\subsection{AR 675}

AR 675 emerged at N12E40 on 2013 February 16 at around 12:00 UT. Several flares, including an M1.9 flare, were observed on February 17 and 18, that is, during the first $48 \mathrm{hr}$ after the AR appearance. Table 1 reports the complete list of flares that occurred in the AR, including the start, peak, and end time, as well as the GOES class of each flare.

The AR emerged as a complex system with several pores characterized by a field of mixed polarity. During its passage across the solar disk, the AR evolved significantly and was characterized by a strong asymmetry between the preceding and following main sunspots. The newly emerging preceding pores displayed a coalescence motion resulting in the formation of a compact leading sunspot, surrounded by a single penumbra. In contrast, the following polarity is seen as a system of individual small pores. Furthermore, a third compact group of pores emerged between the leading sunspot and the following system of pores (see Figures 3(A)-(D)). During the evolution of the AR, the group of compact pores developed a more elongated structure and migrated toward the preceding sunspot (see Figures 3(B) and (C)). The two structures eventually merged by the end of February 18, when no further flaring activity was registered.

Figures 3(E)-(H) show the magnetic field configuration of AR 675 at different times. It is evident from Figure 3(E) that the AR displays a quite complex magnetic field configuration, characterized by several alternating polarities. In particular, at this position, we notice the presence of a compact bipolar 

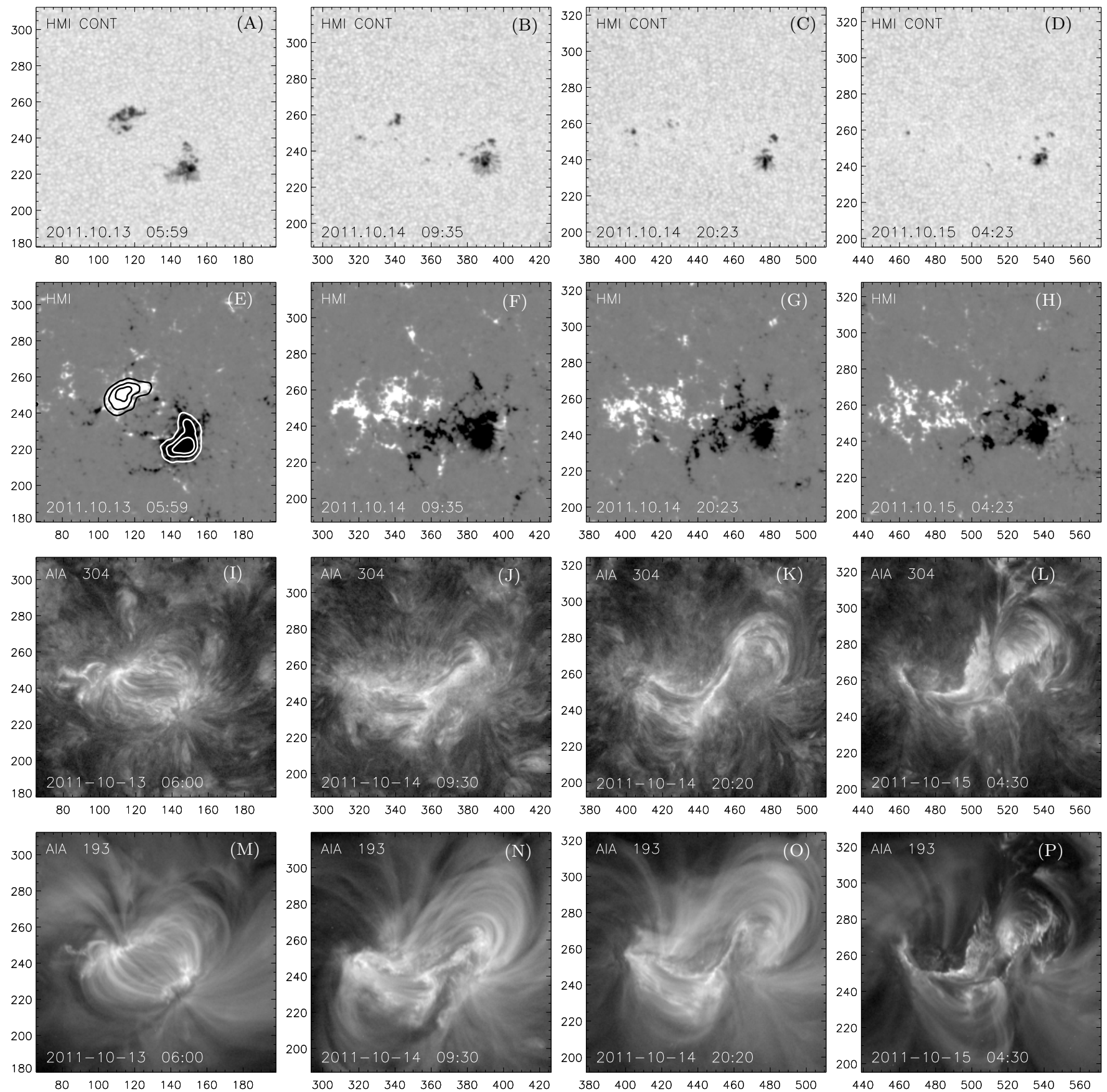

Figure 1. Sequence of images of AR 318 taken at different times: HMI continuum intensity (top), HMI LOS magnetograms (middle top), AIA $304 \AA$ (middle bottom), and AIA $193 \AA$ (bottom). The contours in Figure (E) are $\pm 200, \pm 500$, and $\pm 800 \mathrm{G}$, while the gray scale is saturated at $\pm 300 \mathrm{G}$. North is at the top of the images and west is to the right.

Table 1

C- and M-class Flares that Occurred in AR 675

\begin{tabular}{lcc}
\hline \hline Day & $\begin{array}{c}\text { Time (UT) } \\
\text { (Start, Peak, End) }\end{array}$ & GOES Class \\
\hline 2013 Feb 17 & $00: 31,00: 36,00: 38$ & C1.0 \\
2013 Feb 17 & $15: 26,15: 40,15: 43$ & C2.5 \\
2013 Feb 17 & $15: 45,15: 50,15: 52$ & M1.9 \\
2013 Feb 17 & $19: 57,20: 00,20: 02$ & C1.0 \\
2013 Feb 18 & $02: 36,02: 41,02: 44$ & C1.0 \\
\hline
\end{tabular}

magnetic structure-associated with the compact group of pores-embedded in a larger scale bipolar magnetic field associated with the preceding compact sunspot and the trailing pores (see the arrow in Figure 3(E)). During the evolution of the $\mathrm{AR}$, the compact central dipole further expands (Figure 3(F)) and develops a more complex configuration. The positive polarity of the compact dipole fragments and two positive polarities, separated by a negative magnetic field concentration, are observed (Figure 3(G)). By the end of February 18, the westernmost positive magnetic flux of the compact dipole has been canceled and the AR displays a more clear bipolar configuration. 

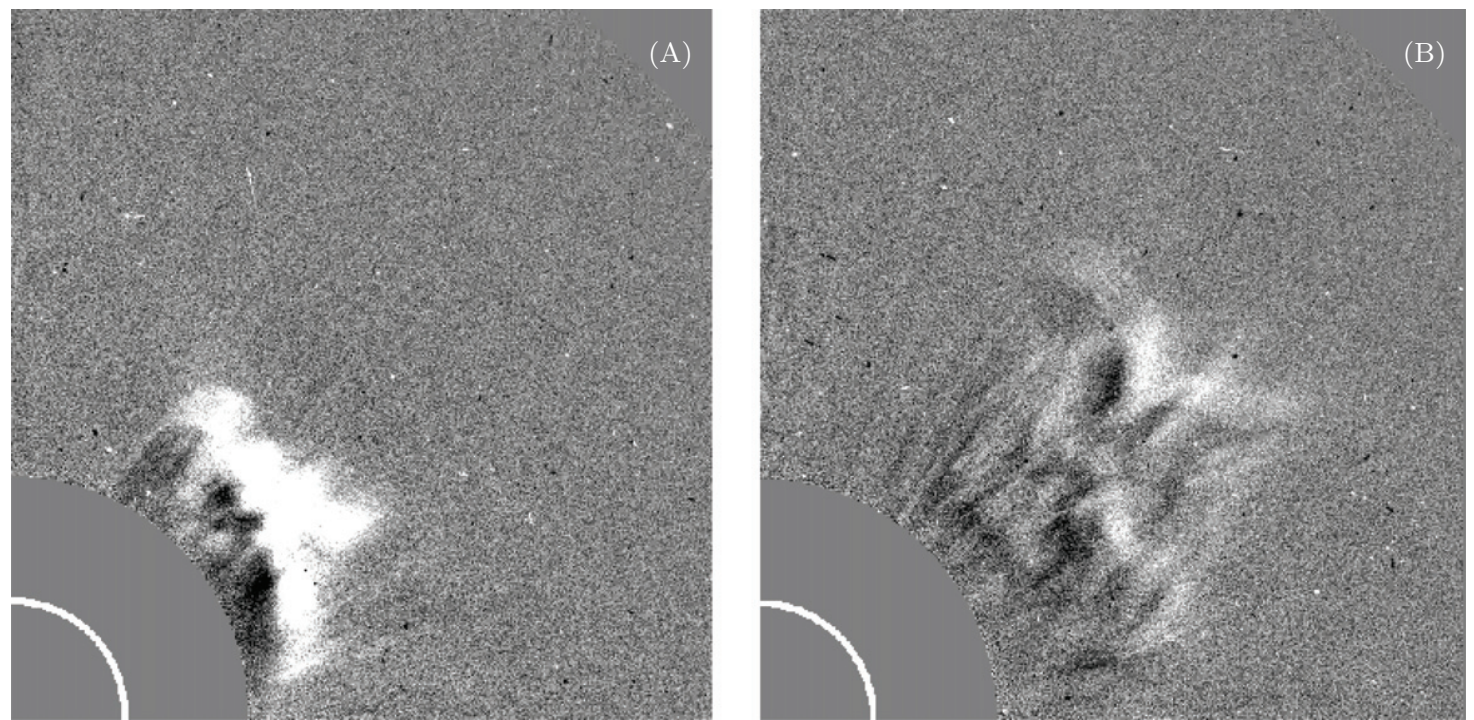

Figure 2. Running difference images obtained by LASCO WL images taken on 2011, October 15 at 5:48 UT (A) and at 6:36 UT (B). The FOV shows the corona at the heliographic distance between 1.5 and 6 solar radii and the position angle between $270^{\circ}$ and $360^{\circ}$.

The chromospheric and coronal evolution of the AR around the time of the major flare as seen in the AIA $304 \AA$ and $193 \AA$ passbands is shown in the two bottom rows of Figure 3. About $12 \mathrm{hr}$ before the filament eruption, i.e., on February 17 at 04:40 UT, a system of loops connecting the negative polarity of the preceding sunspot to the positive flux of the easternmost group of pores is observed. Beneath these overlying loops, we notice the presence of highly sheared loops that probably connect the compact central dipole with the main preceding (negative) and following (positive) polarities of the AR (see the arrows in Figure 3(M)). The images taken about $1 \mathrm{hr}$ before the M1.9 flare clearly show the presence of a filament with its western foot point anchored close to the location of the M1.9 flare, i.e., close to the position of the compact dipole (see the arrows in Figures 3(F), (J), and $(\mathrm{N})$ ). The forward-S shape of this filament suggests that this AR, in this phase, is characterized by positive magnetic helicity, too. Figures $3(\mathrm{~K})-(\mathrm{O})$ show the occurrence of the M1.9 flare and the partial filament eruption. As can be deduced from the accompanying online movie at 171 and $335 \AA$ most of the filament material fell back on the Sun. Only a very minor outflow is visible in the running difference images obtained by COR1-B (see Figures 4(A) and (B)). The configuration of the AR after the M1.9 flare and the partial eruption is shown in Figures $3(\mathrm{~L})-(\mathrm{P})$. As can be seen from these figures, the filament is still visible. This suggests that the partial eruption was associated with a portion of the filament, probably located above the PIL of the compact dipole. Finally, we would like to stress that, unlike AR 318, no sigmoid is observed during the evolution of AR 675 .

The magnetic configuration inferred from SHARP data clearly indicates the presence of a bipolar structure in between the two main polarities of the AR, underlying the filament observed in the upper atmospheric layers. Along the PIL, between the opposite polarities of this bipole, there are points where the horizontal magnetic field is strong $(\sim 1500 \mathrm{G})$ and parallel to the PIL. We have calculated the shear between the observed (measured) horizontal field and the horizontal field derived through a potential field extrapolation (Wang et al. 1994), computed using the method described by Alissandrakis (1981). Following the procedure of Falconer et al. (2002) and Jiang et al. (2014), we estimated the shear by using the following horizontal shear angle equation (Gosain \& Venkatakrishnan 2010):

$$
\theta=\arccos \frac{\mathbf{B}_{\mathbf{h}}^{\text {obs }} \cdot \mathbf{B}_{\mathbf{h}}^{\text {pot }}}{\left|B_{h}^{\text {obs }}\right|\left|B_{h}^{\text {pot }}\right|},
$$

where $B^{\text {pot }}$ is the unique potential field such that $B_{r}^{\text {pot }}=B_{r}^{\text {obs }}$. We have also computed the dip angle, which measures the difference between the inclination angle of the observed field and that of the potential field (see, e.g., Gosain \& Venkatakrishnan 2010; Petrie 2012). This is defined as

$$
\Delta \gamma=\gamma^{\mathrm{obs}}-\gamma^{\mathrm{pot}}
$$

where $\gamma=90^{\circ}-\arctan \left(B_{r} / B_{h}\right)$ is the inclination angle derived in both cases. The resulting maps are shown in Figure 5, where we show the evolution of the shear and dip angles before and after the M-class flare. We can see that the region between the opposite polarities of the compact bipole underlying the filament is characterized by high values of the shear angle, larger than $45^{\circ}$ and seems to increase after the flare, according to Wang et al. (1994; see the arrows in the left panels of Figure 5). The dip angle exhibits a similar behavior, as it increases after the flare, in particular in the positive patch of the bipole (see the arrows in the right panels of Figure 5). Finally, we note that the regions of the FOV far from the PIL that show a large shear angle, higher than $100^{\circ}$, may be affected by a possible error in the $180^{\circ}$ azimuth ambiguity resolution.

\section{MAGNETIC HELICITY ACCUMULATION}

Considering the radial component of the HMI SHARPs data with a field of view of $243^{\prime \prime} \times 94^{\prime \prime}(484 \times 187$ pixels $)$ and $251^{\prime \prime} \times 92^{\prime \prime}(500 \times 183$ pixels $)$ centered on AR 318 and AR 675, respectively, we measured the temporal evolution of the positive, negative, and unsigned magnetic fluxes for AR 318 and AR 675 . In Figures 6(A) and (B), we report the measured magnetic fluxes with corresponding error bars. We estimated the errors on the magnetic flux by propagating the experimental errors and considering the HMI sensitivity of $10 \mathrm{G}$ (Schou et al. 2012). 

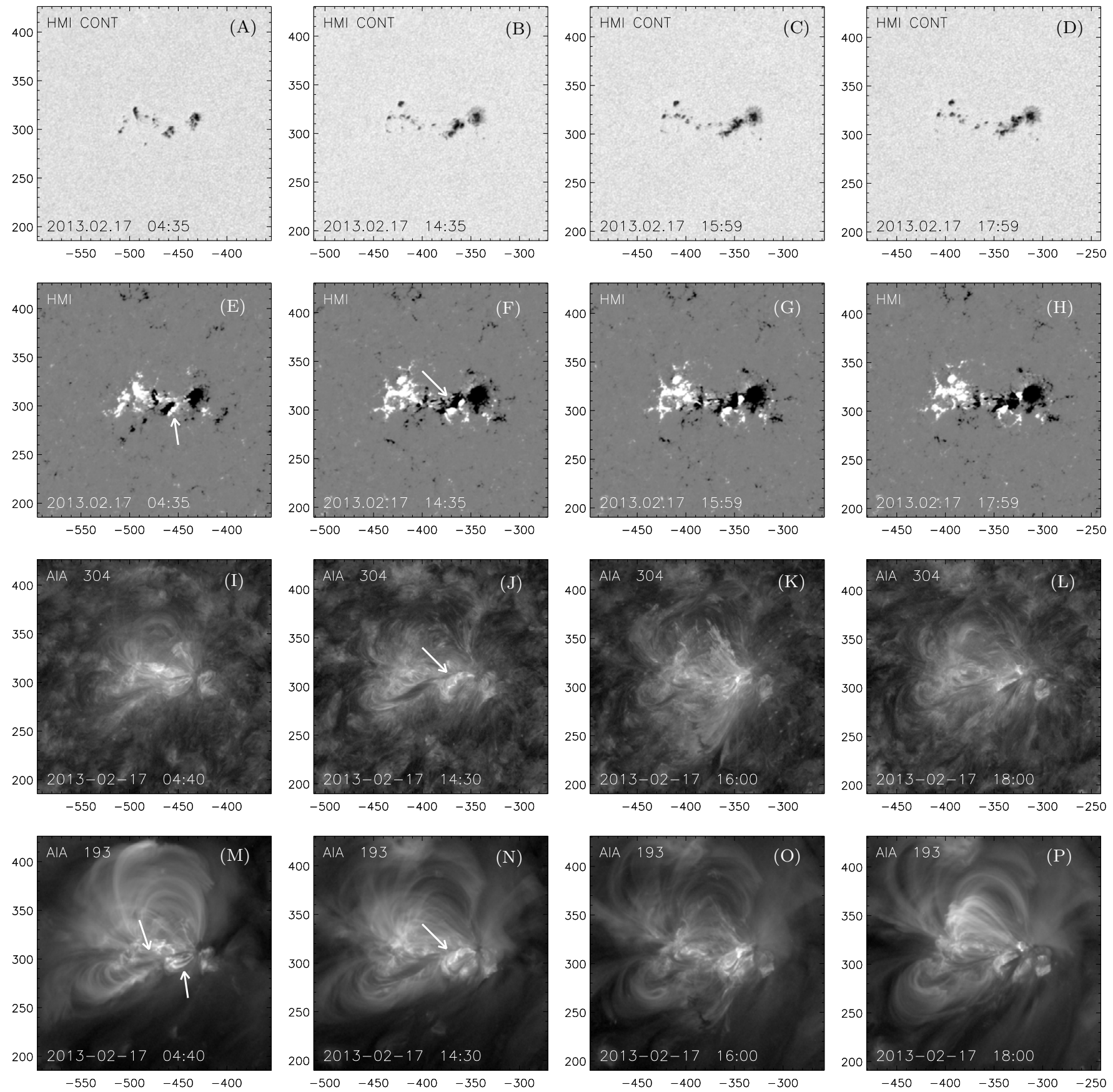

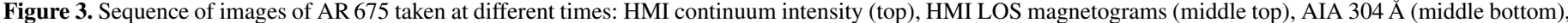

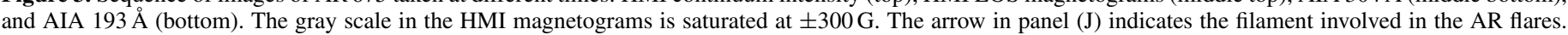
North is at the top of the images and west is to the right.

(An animation of this figure is available in the online journal.)

A similar behavior has been observed for both ARs: a phase of emergence during the first 30-40 hr of observations and a subsequent decay phase until $80 \mathrm{hr}$ from the beginning of the observation time interval. A further small emergence of magnetic flux is also observed near the end of the observations in AR 318. The maximum strength of the unsigned magnetic flux of the AR $675\left(5.8 \times 10^{21} \mathrm{Mx}\right)$ is about two times that of the AR $318\left(2.8 \times 10^{21} \mathrm{Mx}\right)$. For both ARs, the magnetic flux of the two polarities is not balanced during the decay phase. Soon after the main emergence phase, a high negative flux excess-about two times greater than the positive flux-is measured for both ARs. This imbalance might be due to the greater compactness of the preceding negative sunspot with respect to the following fragmented group of pores. This is in contrast to other possibilities of flux imbalance, such as transequatorial magnetic connectivities, as suggested in Choudhary et al. (2002). It is worth noting that the C2.3 class flare occurred only at the end of the decay phase for AR 318, while the $\mathrm{C}$ - and M-class flares occurred during the phase of emergence or around the maximum of magnetic flux strength for AR 675 .

To compute the magnetic helicity flux through the photosphere, we determined the mean magnetogram corresponding to the average between two consecutive magnetograms and we measured the horizontal velocity fields by means of the 

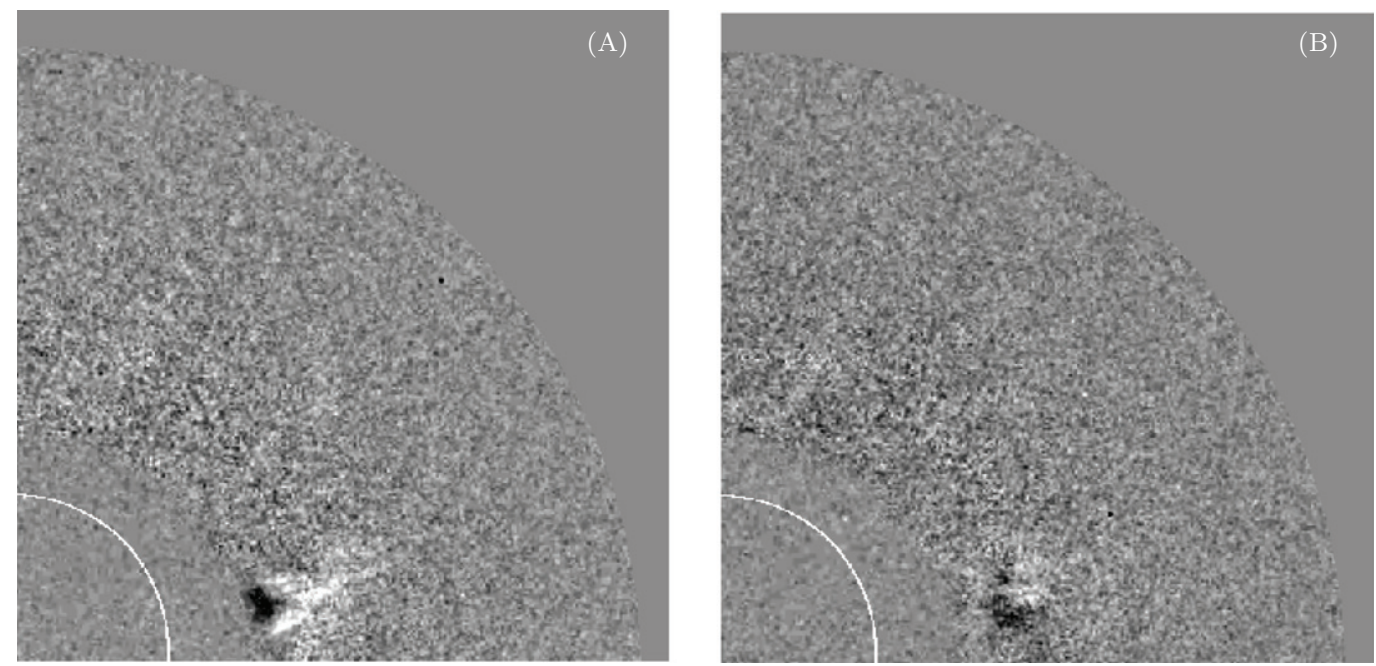

Figure 4. Running difference images obtained by STEREO COR1-B images taken on 2013 February 17 at 16:30 UT (A) and at 16:50 UT (B). The FOV shows the corona at the heliographic distance between 1.4 and 4 solar radii and the position angle between $270^{\circ}$ and $360^{\circ}$.
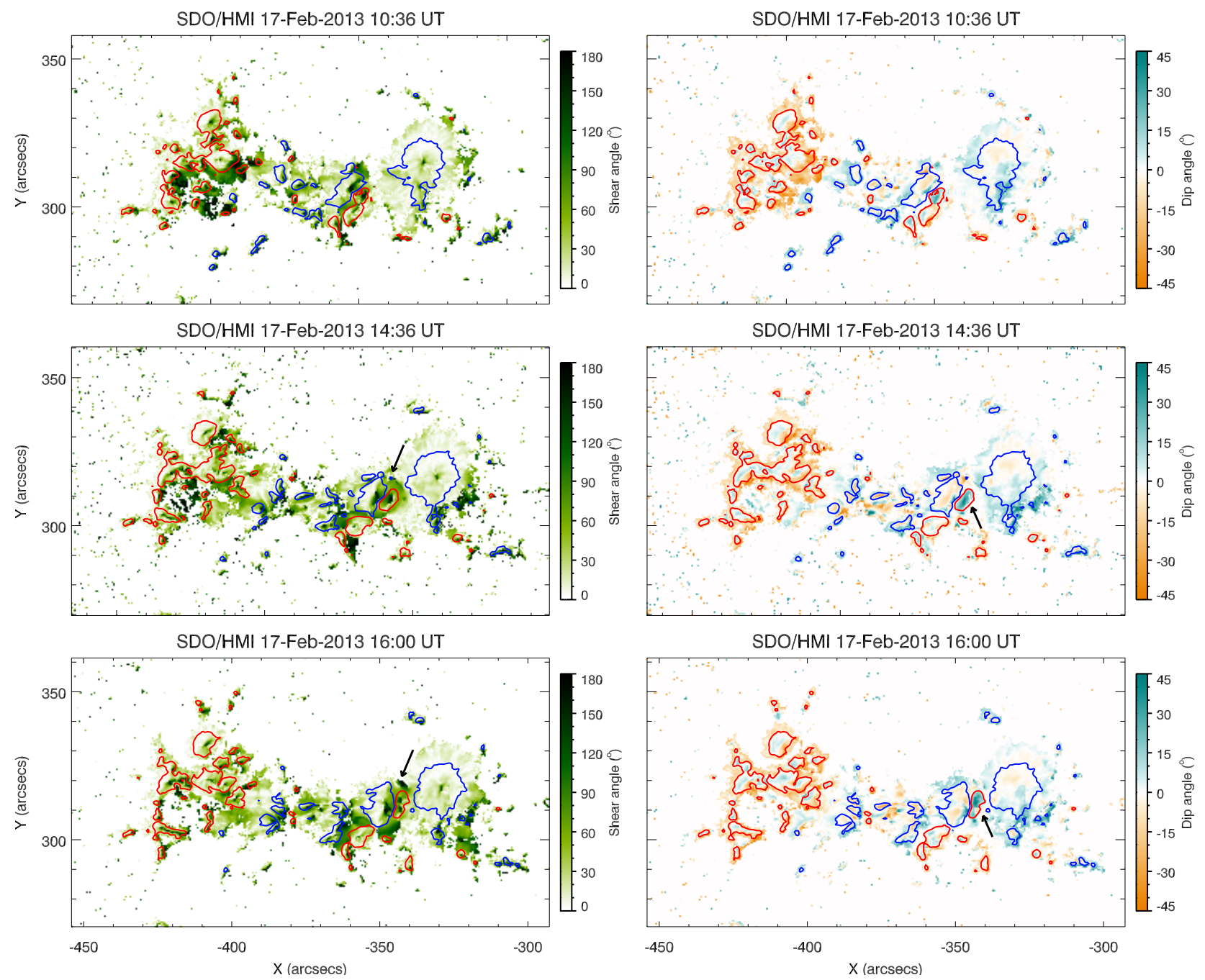

Figure 5. Evolution of the shear angle (left panels) and the dip angle (right panels) between the measured field and the field derived using the potential extrapolations. The white background includes points with total fields lower than $200 \mathrm{G}$ that are not represented. The red (blue) contours indicate a longitudinal field of $+500 \mathrm{G}$ $(-500 \mathrm{G})$. The arrows in the left panels indicate the compact bipole underlying the filament, while the arrows in the right panels indicate the positive patch of the same bipole.

(A color version of this figure is available in the online journal.) 

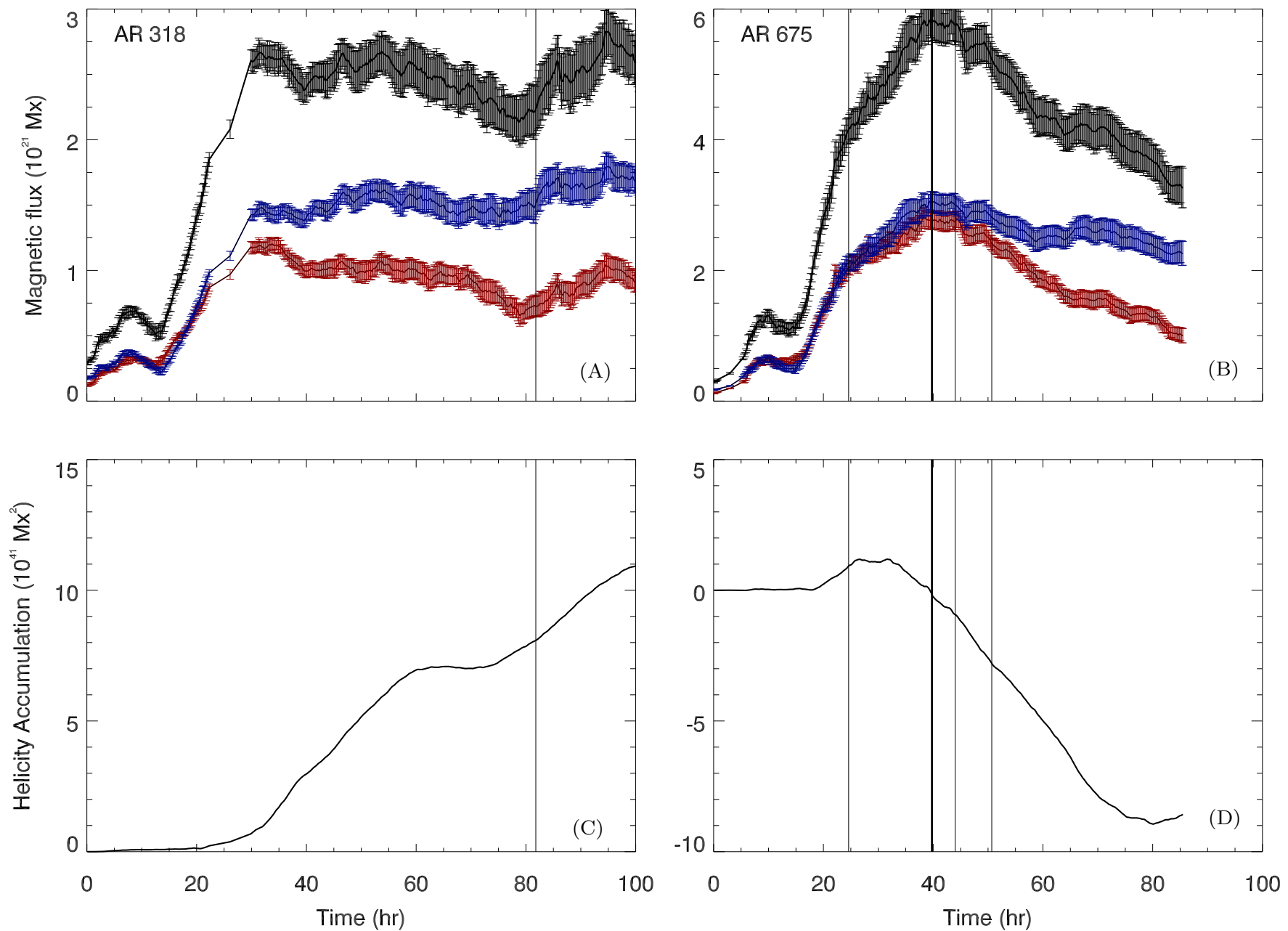

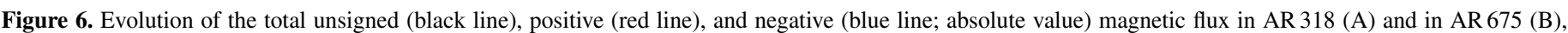

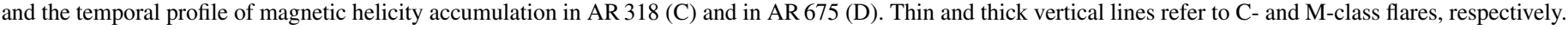
Time $t=0 \mathrm{hr}$ corresponds to 2011 October 11 at 19:12 UT and to 2013 February 16 at 00:00 UT for AR 318 and AR 675, respectively.

(A color version of this figure is available in the online journal.)

differential affine velocity estimator (DAVE) technique (Schuck $2005,2006)$, using a window size of the apodizing window of 11 pixels $\left(55^{\prime \prime} 5\right)$. Then, we estimated the magnetic helicity flux for both ARs using Equation (18) of Pariat et al. (2005):

$$
G_{\vartheta}=-\frac{B_{n}}{2 \pi} \int_{S^{\prime}} \frac{d \vartheta(\mathbf{r})}{d t} B_{n}^{\prime} d S^{\prime}
$$

where $\mathbf{r}$ is the vector between two photospheric points $x$ and $x^{\prime}$ and, consequently, $(d \vartheta(\mathbf{r}) / d t)$ is the relative rotation rate of these points, $B_{n}$ and $B_{n}^{\prime}=B_{n}\left(x^{\prime}\right)$ are the normal components of the photospheric magnetic field, and $S^{\prime}$ is the integration surface. In our case, we used the radial component of the HMI vector magnetograms as $B_{n}$ and $B_{n}^{\prime}$. Finally, we computed the magnetic helicity accumulation in both ARs since the beginning of the observations.

The magnetic helicity accumulation in AR 318 is reported in Figure 6(C), from which it is possible to infer that the magnetic helicity remains close to zero for almost $20 \mathrm{hr}$ (i.e., until the magnetic flux shows the abrupt increase). Later on, the first rapid increase of positive helicity is observed for about $30 \mathrm{hr}$, then a flat phase occurs and, about $10 \mathrm{hr}$ before the $\mathrm{C} 2.3$ flare, a second, more moderate, increase phase takes place. The maximum value reached by the magnetic helicity accumulation is $1.1 \times 10^{42} \mathrm{Mx}^{2}$. Note that no change in the magnetic helicity accumulation is observed after the flare/CME occurrence. On the basis of the results obtained applying DAVE to HMI magnetograms, we could also deduce that, during the phase of rapid increase in the magnetic helicity accumulation, the horizontal velocity field distribution shows a counterclockwise rotation of the bipole axis, as well as a separation between the two magnetic polarities. This motion corresponds to a positive helicity flux over the whole AR, as shown in the helicity flux density map reported in the right panel of Figure 7.

In AR 675, during the initial phase of flux emergence, no significant magnetic helicity is injected into the corona through the photosphere, as reported in the plot of Figure 6(D). However, about $18 \mathrm{hr}$ after the observations began, positive magnetic helicity is injected for about $10 \mathrm{hr}$. This coincides with the sudden increase of magnetic flux observed in Figure 6(B). After about $32 \mathrm{hr}$ from the beginning of the observations, a change in the trend of helicity accumulation is recorded and negative magnetic helicity is injected into the corona. During this phase, the horizontal velocity fields, inferred from the application of DAVE to HMI magnetograms, show a persistent convergence motion of the compact dipole toward the preceding negative polarity. As a result of this evolution, part of the positive magnetic flux of the compact bipole is canceled and 

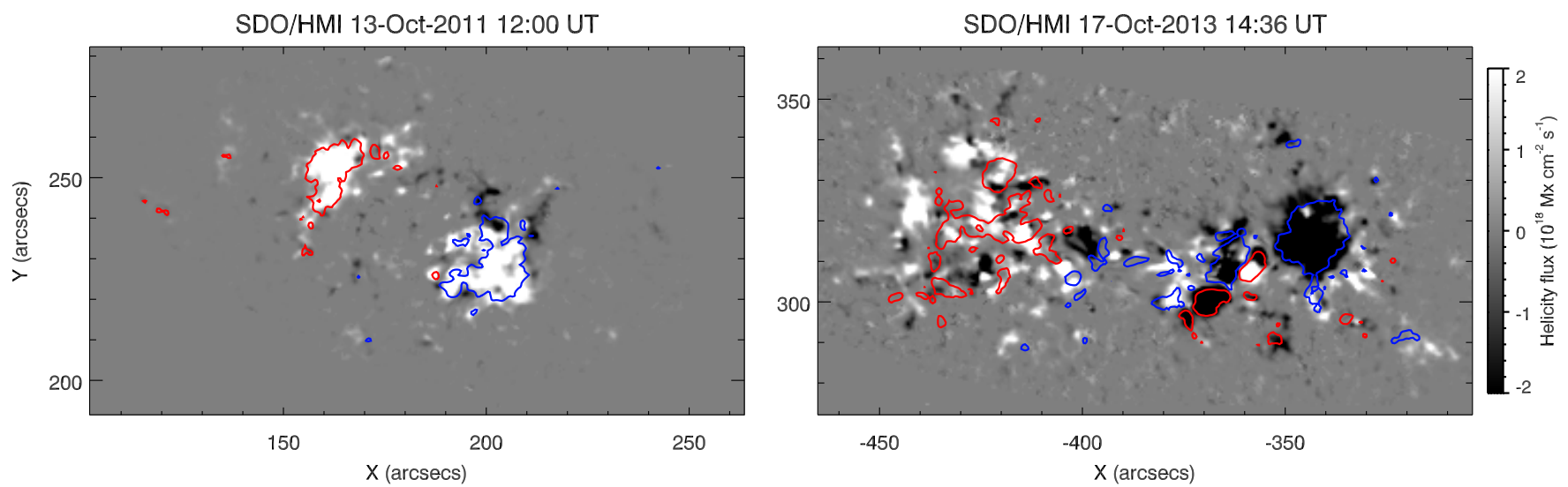

Figure 7. Helicity flux density maps of AR 318 (left panel) and AR 675 (right panel) computed from Equation (3). The red (blue) contours indicate a longitudinal field of $+500 \mathrm{G}(-500 \mathrm{G})$.

(A color version of this figure is available in the online journal.)

Table 2

AR Characteristics

\begin{tabular}{lcc}
\hline \hline Parameter & NOAA 11318 & NOAA 11675 \\
\hline AR classification & $\beta \gamma$ & $\beta \gamma$ \\
Time of start of flux emergence & $17: 30$ UT on Oct 12 & $12: 00$ UT on Feb 16 \\
Time of filament eruption & $04: 19$ UT on Oct 15 & $15: 50$ UT on Feb 17 \\
Maximum unsigned magnetic flux (Mx) & $2.8 \times 10^{21}$ & $5.8 \times 10^{21}$ \\
Magnetic flux imbalance & $\Phi_{(-)} \sim 2 \times \Phi_{(+)}$ & $\Phi_{(-)} \sim 2 \times \Phi_{(+)}$ \\
S-shape & Forward & Forward \\
Hemispheric helicity rule & No & Partially \\
Flare associated with filament eruption & C2.3 & M1.9 \\
Flare energy (W) & $1.4 \times 10^{-3}$ & $2.9 \times 10^{-3}$ \\
CME & Yes & NO \\
Helicity accumulation before the flare $\left(\mathrm{Mx}^{2}\right)$ & $\sim 8.0 \times 10^{41}$ & $\sim 0$ \\
Prevalent sign of helicity flux in the AR & Positive & Negative \\
\hline
\end{tabular}

Note. $\Phi_{(-)}$and $\Phi_{(+)}$indicate the negative and positive magnetic flux, respectively.

a significant negative helicity is injected into the corona, as shown in the right panel of Figure 7, where we can see that in the emerging bipole the negative helicity flux prevails. This trend of helicity accumulation continues up to the end of the observations. Interestingly, the accumulated helicity returned to zero when the M1.9 flare occurred.

\section{DISCUSSION AND CONCLUSIONS}

The comparison between the two targets studied in this paper allows us to discuss some implications related to the magnetic helicity and the FR emergence and eruption. We summarized all of the results in Table 2 .

In AR 318, the emergence of the FR in the corona is documented by the transition of the main loop system visible at $193 \AA$ from an arcade-like to a sigmoid-like configuration. This evolution occurred mainly during October 14 and corresponds to the phase of higher flux imbalance between the two polarities and to the plateau in the accumulation of the magnetic helicity, i.e., between $t=60$ and $t=70 \mathrm{hr}$.

For AR 675, the formation of the FR is not as clear as it is in AR 318. However, we can speculate that the FR forms between $t=20$ and $t=40 \mathrm{hr}$, when the main filament of the AR appears visible at $304 \AA$ (see Figures 1(I) and (J)). This filament appears in correspondence with the compact bipolar magnetic structure embedded in the larger scale pre-existing magnetic field of the AR (see Figures 1(E) and (F)). This region is also characterized by a high value of the shear, as shown by the comparison between the observed vector components of the magnetic field and the retrieved potential configuration (Figure 5). We note that the phase of emergence of the compact bipolar magnetic structure starts on February 16 at about 17:30 UT ( $t=17.5 \mathrm{hr}$ in Figure 6(B)), when we observe the steep increase of the magnetic flux in the whole AR. This phase, preceding the M1.9 flare, corresponds to a bump in the magnetic helicity accumulation (Figure 6(D)). We also note that the flip of the sign in the magnetic helicity accumulation before the flare corresponds to the time interval when the bipole is fully emerged and starts to migrate toward the preceding sunspot.

Although both ARs are located in the northern hemisphere, their magnetic helicity flux does not fully obey the general cycleinvariant hemispheric helicity rule (Seehafer 1990; Pevtsov et al. 1995), because they are positive during the whole observation time interval for AR 318 and until just before the M1.9 flare for the AR 675. The sign of the magnetic helicity is also confirmed by the forward-S shape sigmoid in the AR 318 and by the forward-S shape filament in the AR 675. Therefore, according to Canfield et al. (2007), they do not conform to the helicity hemispheric pattern. We notice that, while AR 318 accumulates a positive magnetic helicity of about $1.1 \times 10^{42} \mathrm{Mx}^{2}$ before the $\mathrm{C} 2.3$ flare, the budget of the magnetic helicity accumulated by AR 675 before the M1.9 flare is about zero. It is also worth noting that the peak of the accumulated helicity for the AR 318 is delayed with respect to the peak of the flux emergence 
by a few days, in agreement with the model of Longcope \& Welsch (2000).

From the helicity flux maps (see Figure 7), it is also clear that the negative helicity dominates in AR 675, because its leader spot has negative helicity flux, while following dispersed polarity has mixed signs. This is different from AR 318, in which both polarities are rather compact and characterized by positive helicity flux.

The different activity registered in the two ARs sheds light on the role played by the surrounding magnetic field in the eruption of an FR. In fact, AR 318 was characterized by a simple bipole configuration at photospheric level and the only C2.3 flare was associated with a CME, which probably carried away the magnetic helicity accumulated in the corona. Instead, in spite of its photospheric complexity, AR 675 showed several small flares before the main one of $\mathrm{M}$ class, but no CME occurred: only a faint and small emission was observed by STEREO/COR1 after the M flare, while LASCO and STEREO/COR2 did not observe any event temporally correlated with this AR. The fact that AR 675 does not show any significant eruptive event and that the M1.9 flare can be considered as an almost confined flare may be ascribed to the presence of an overlying magnetic field. In fact, according to Kliem \& Torök (2006), in the case of AR 675, we think that the overlying magnetic field exerts a constraining force that inhibits the solar eruption. Moreover, the B- and C-class flares preceding the main event may be interpreted as signatures of magnetic reconnection processes between the emerging FR and the overlying magnetic flux system that may dissipate the magnetic free energy and helicity from the FR into the ambient field, reducing the amount of energy available for the eruption.

Therefore, our results highlight different conditions for the occurrence of flares and/or CMEs. It is not enough that an AR accumulates magnetic helicity in the corona for the occurrence of a CME to take place: in this study, both ARs accumulated more or less the same amount of magnetic helicity during the same observing time interval, but only one of the ARs, the simplest one at the photospheric level, produced a CME. Instead, several flares occurred during the earlier phase of observations in the more complex AR, but these events did not give rise to similar eruptive events in the outer corona. This allows us to speculate that for the occurrence of CMEs associated with ARs, not only is the presence of an FR important, but also the configuration of the surrounding magnetic field. Recently, several simulations showed that solar eruptions may occur when two magnetic flux systems have opposite signs of magnetic helicity (Kusano et al. 2004), or when they are antiparallel (Galsgaard et al. 2007), or when the inward magnetic tension of the external field is not able to balance the outward magnetic pressure of the FR (Kliem \& Torök 2006). We conclude that the two cases reported in this study provide evidence that the photospheric complexity of an AR is only a partial indication of the real likelihood for an AR to produce CMEs. However, more statistics, taking into account more observations with high spatial and temporal resolution, are necessary to more comprehensively address the role of helicity accumulation and AR complexity in the likelihood for an AR to produce CMEs.

The research leading to these results has received funding from the European Commission's Seventh Framework
Programme under the grant agreements eHEROES (project No. 284461), F-Chroma (project No. 606862), and SOLARNET (project No. 312495). This research is partly supported by the Italian MIUR-PRIN grant 2012P2HRCR on "The Active Sun and Its Effects on Space and Earth Climate" and by Space Weather Italian COmmunity (SWICO) Research Program. The contribution of F.P.Z. has been funded by the FWO Vlaanderen through grant agreement No. 1272714N (FWO Vlaanderen).

Facility: SDO (HMI, AIA)

\section{REFERENCES}

Alissandrakis, C. E. 1981, A\&A, 100, 197

Archontis, V., \& Hood, A. W. 2010, A\&A, 514, A56

Archontis, V., Hood, A. W., \& Tsinganos, K. 2014, ApJL, 786, L21

Berger, M. A., \& Field, G. B. 1984, JFM, 147, 133

Bobra, M. G., Sun, X., Hoeksema, J. T., et al. 2014, SoPh, 289, 3549

Borrero, J. M., Tomczyk, S., Kubo, M., et al. 2011, SoPh, 273, 267

Brown, D. S., Nightingale, R. W., Alexander, D., et al. 2003, SoPh, 216, 79

Canfield, R. C., Kazachenko, M. D., Acton, L. W., et al. 2007, ApJ, 671, 81

Choudhary, D. P., Venkatakrishnan, P., \& Gosain, S. 2002, ApJ, 573, 851

Démoulin, P., \& Pariat, E. P. 2009, AdSpR, 43, 1013

Emonet, T., \& Moreno-Insertis, F. 1998, ApJ, 492, 804

Falconer, D. A., Moore, R. L., \& Gary, G. A. 2002, ApJ, 569, 1016

Fan, Y. 2009, ApJ, 697, 1529

Fan, Y., Zweibel, E. G., \& Lantz, S. R. 1998, ApJ, 493, 480

Galsgaard, D., Archontis, V., Moreno-Insertis, F., \& Hood, A. W. 2007, ApJ, 666,516

Gary, G. A., \& Hagyard, M. J. 1990, SoPh, 126, 21

Gibson, S. E., Fan, Y., Mandrini, C., Fisher, G., \& Demoulin, P. 2004, ApJ, 617,600

Gosain, S., \& Venkatakrishnan, P. 2010, ApJL, 720, L137

Hoeksema, J. T., Liu, Y., Hayashi, K., et al. 2014, SoPh, 289, 3483

Hood, A. W., Archontis, V., Galsgaard, K., \& Moreno-Insertis, F. 2009, A\&A, 503,999

Jiang, C., Wu, S. T., Feng, X., \& Hu, Q. 2014, ApJ, 780, 55

Kaiser, M. L., Kucera, T. A., Davila, J. M., et al. 2008, SSRv, 136, 5

Kliem, B., \& Torök, T. 2006, PhRvL, 96, 255002

Kusano, K., Maeshiro, T., Yokoyama, T., \& Sakurai, T. 2004, ApJ, 610, 537

Leka, K. D., Canfield, R. C., McClymont, A. N., \& van Driel-Gesztelyi, L. 1996, ApJ, 462, 547

Lemen, J. R., Title, A. M., Akin, D. J., et al. 2012, SoPh, 275, 17

Lites, B. W., Low, B. C., Martinez-Pillet, V., et al. 1995, ApJ, 446, 877

Longcope, D. W., \& Welsch, B. T. 2000, ApJ, 545, 1089

Low, B. C. 1996, SoPh, 167, 217

Luoni, M. L., Démoulin, P., Mandrini, C. H., \& van Driel-Gesztelyi, L. 2011, SoPh, 270, 45

Magara, T. 2006, ApJ, 653, 1499

Metcalf, T. R. 1994, SoPh, 155, 235

Pariat, E., Démoulin, P., \& Berger, M. A. 2005, A\&A, 439, 1191

Pesnell, W. D., Thompson, B. J., \& Chamberlin, P. C. 2012, SoPh, 275, 3

Petrie, G. J. D. 2012, ApJ, 759, 50

Pevtsov, A. A., Canfield, R. C., \& Metcalf, T. R. 1995, ApJL, 440, L109

Rust, D. 1994, GeoRL, 21, 241

Rust, D., \& Kumar, A. 1994, SoPh, 155, 69

Schmieder, B., Demoulin, P., \& Aulanier, G. 2013, AdSpR, 51, 1967

Schou, J., Scherrer, P. H., Bush, R. I., et al. 2012, SoPh, 275, 229

Schuck, P. W. 2005, ApJL, 632, L53

Schuck, P. W. 2006, ApJ, 646, 1358

Seehafer, N. 1990, SoPh, 125, 219

Spadaro, D., Billotta, S., Contarino, L., Romano, P., \& Zuccarello, F. 2004, A\&A, 425, 309

Sun, X. 2013, arXiv:1309.2392

Tanaka, K. 1991, SoPh, 136, 133

Thompson, W. T., Davila, J. M., Fisher, R. R., et al. 2003, Proc. SPIE, 4853, 1

Thompson, W. T., \& Reginald, N. L. 2008, SoPh, 250, 443

Wang, H., Ewell, M. W. J., Zirin, H., \& Ai, G. 1994, ApJ, 424, 436

Zuccarello, F. P., Meliani, Z., \& Poedts, S. 2012, ApJ, 758, 117 